\title{
Hippocampal glucose uptake as a surrogate of metabolic change of microglia in Alzheimer's disease
}

Hongyoon Choi ${ }^{1,2^{*}+}$, Yoori Choi ${ }^{1,3+}$, Eun Ji Lee ${ }^{1,3}$, Hyun Kim ${ }^{1,3}$, Youngsun Lee ${ }^{1,3}$, Seokjun Kwon ${ }^{1,3}$, Do Won Hwang ${ }^{1,3}$, Dong Soo Lee ${ }^{1,2,3^{*}}$ and for the Alzheimer's Disease Neuroimaging Initiative

\begin{abstract}
Background: Dynamically altered microglia play an important role in the progression of Alzheimer's disease (AD). Here, we found a close association of the metabolic reconfiguration of microglia with increased hippocampal glucose uptake on $\left[^{18} \mathrm{~F}\right]$ fluorodeoxyglucose (FDG) PET.
\end{abstract}

Methods: We used an AD animal model, 5xFAD, to analyze hippocampal glucose metabolism using both animal FDG PET and ex vivo FDG uptake test. Cells of the hippocampus were isolated to perform single-cell RNAsequencing (scRNA-seq). The molecular features of cells associated with glucose metabolism were analyzed at a single-cell level. In order to apply our findings to human brain imaging study, brain FDG PET data obtained from the Alzheimer's Disease Neuroimaging Initiative were analyzed. FDG uptake in the hippocampus was compared according to the diagnosis, AD, mild cognitive impairment, and controls. The correlation analysis between hippocampal FDG uptake and soluble TREM2 in cerebrospinal fluid was performed.

Results: In the animal study, 8- and 12-month-old 5xFAD mice showed higher FDG uptake in the hippocampus than wild-type mice. Cellular FDG uptake tests showed that FDG activity in hippocampal microglia was increased in the AD model, while FDG activity in non-microglial cells of the hippocampus was not different between the AD model and wild-type. scRNA-seq data showed that changes in glucose metabolism signatures including glucose transporters, glycolysis and oxidative phosphorylation, mainly occurred in microglia. A subset of microglia with higher glucose transporters with defective glycolysis and oxidative phosphorylation was increased according to disease progression. In the human imaging study, we found a positive association between soluble TREM2 and hippocampal FDG uptake. FDG uptake in the hippocampus at the baseline scan predicted mild cognitive impairment conversion to AD.

\footnotetext{
* Correspondence: chy1000@snu.ac.kr; dsl@plaza.snu.ac.kr

Data used in preparation of this article were obtained from the Alzheimer's

Disease Neuroimaging Initiative (ADNI) database (adni.loni.usc.edu). As such,

the investigators within the ADNI contributed to the design and

implementation of ADNI and/or provided data but did not participate in

analysis or writing of this report. A complete listing of ADNI investigators can

be found at: http://adni.loni.usc.edu/wp-content/uploads/how_to_apply/

ADNI_Acknowledgement_List.pdf

${ }^{\dagger}$ Hongyoon Choi and Yoori Choi contributed equally to this work.

'Department of Nuclear Medicine, Seoul National University Hospital, 101

Daehak-ro, Jongno-Gu, Seoul 03080 Seoul, Republic of Korea

Full list of author information is available at the end of the article
}

\section{$\triangle B M C$}

(c) The Author(s). 2021 Open Access This article is licensed under a Creative Commons Attribution 4.0 International License, which permits use, sharing, adaptation, distribution and reproduction in any medium or format, as long as you give appropriate credit to the original author(s) and the source, provide a link to the Creative Commons licence, and indicate if changes were made. The images or other third party material in this article are included in the article's Creative Commons licence, unless indicated otherwise in a credit line to the material. If material is not included in the article's Creative Commons licence and your intended use is not permitted by statutory regulation or exceeds the permitted use, you will need to obtain permission directly from the copyright holder. To view a copy of this licence, visit http://creativecommons.org/licenses/by/4.0/ The Creative Commons Public Domain Dedication waiver (http://creativecommons.org/publicdomain/zero/1.0/) applies to the data made available in this article, unless otherwise stated in a credit line to the data. 
Conclusions: We identified the reconfiguration of microglial glucose metabolism in the hippocampus of AD, which could be evaluated by FDG PET as a feasible surrogate imaging biomarker for microglia-mediated inflammation.

Keywords: Alzheimer's disease, Microglia, Positron emission tomography, Hippocampus, Single-cell RNAsequencing

\section{Background}

Alzheimer's disease (AD) is the most common neurodegenerative disorder. AD is characterized by neuronal loss causing cognitive decline and the presence of amyloid beta deposits and neurofibrillary tangles in the brain $[1$, 2]. These pathological features serve as biomarkers to diagnose $\mathrm{AD}$ and are the basis for the ATN framework, a biomarker-based research (A, amyloid; T, phosphorylated tau; N, neurodegeneration) [3]. Although the stage of the AD continuum is diagnosed through ATN biomarker measurements, other biomarkers are needed to know prognosis and discover treatment targets of $\mathrm{AD}$. Recently, neuroinflammation is not only a major factor with amyloid and tau in the pathology of $\mathrm{AD}$, but also plays an important role in disease progression [4]. In particular, microglia play a key role in neuroinflammation associated with amyloid and tau accumulation, and synaptic dysfunction [5].

These pathologic characteristics are closely linked to the immune reaction, which is a complicated process in which microglia play a crucial role [4]. As microglia-mediated immune reactions are a dynamic process caused by various subtypes of microglia in the brain, recent studies have focused on the evaluation of the diversity of microglia potentially associated with the progression of $\mathrm{AD}$ pathophysiology [6, 7]. In general, reactive microglia play a role in the protection from CNS insults such as $A \beta$. Reactive microglia increase $A \beta$ endocytosis and phagocytosis in $\mathrm{AD}$, but these functions are degenerated due to chronic inflammatory conditions in AD [8]. A persistent inflammation induces changes of their phenotype that exhibits proinflammatory cytokines and nitric oxide release, increased proliferative responses, and decreased neurotrophic factors [9]. During this dynamic microgliamediated inflammation, disease-associated microglia (DAM) have been defined as a subset of microglia that acquire a unique functional signature to attenuate the progression of neuronal loss in neurodegenerative disorders mediated by Triggering receptor expressed on myeloid cells-2 (TREM2) [5, 10]. TREM2 is a key surface receptor for the microglial response to neurodegeneration-related proteins, modulates phagocytosis, lipid metabolism, and metabolic shift to promote cell survival and restrict inflammation [11]. As a biomarker, the soluble form of TREM2 (sTREM2) measured in cerebrospinal fluid (CSF) recently receives attention in $\mathrm{AD}$ and other neurodegeneration disorders [12].
One of the key changes in microglia-mediated inflammation is metabolism, as immune cells depend on various metabolic pathways according to energy demands. The reactivity of the innate immune system is associated with a metabolic shift from oxidative phosphorylation (OXPHOS) to glycolysis, which is a rapid process of energy production. These active metabolic processes can be suppressed by chronic inflammatory processes due to broad defects in energy metabolism that underlie immunoparalysis [13]. This immunoparalysis induces phagocytic dysfunction of microglia in $\mathrm{AD}[14,15]$.

Because of the important role of microglia in $\mathrm{AD}$, a search for a biomarker for the functional evaluation of microglia has been attempted. In particular, molecular imaging methods based on positron emission tomography (PET) have been developed for targeting microglia. For example, $\left[{ }^{11} \mathrm{C}\right] \mathrm{PK} 11195$ (1-[2-chlorophenyl]-Nmethyl-N-[1-methyl-propyl]-3-isoquinolinecarboxamide) binds to translocator protein- $18 \mathrm{kDa}$ (TSPO), a marker of reactive microglia, has been used to evaluate microglial density in the brain [16, 17]. Although several modified radiotracers targeting TSPO have been recently studied, one of the limitations of TSPO PET includes the difficulty in reflecting recently emerging microglial reprogramming of dynamic changes according to neurodegeneration. Meanwhile, fluorodeoxyglucose (FDG), the most commonly used radiotracer for PET imaging, has been used to evaluate metabolic patterns of the brain in AD [18, 19]. As FDG PET represents regional glucose metabolism which is a universal feature of all cells, it is intrinsically difficult to differentiate the origin of FDG uptake and whether hypermetabolism on PET results from neurons or glia $[20,21]$. For human $\mathrm{AD}$ studies, decreased glucose metabolism is mainly identified in some specific brain regions including the posterior cingulate, superior parietal and lateral temporal cortices $[18,19]$. Although the results are controversial, a few studies have shown increased glucose metabolism in the hippocampus of $\mathrm{AD}$ [22-25]. However, the reason for regional differences in neuronal loss and metabolic change is still unknown, where neuronal loss is typically found in the hippocampus, and hypometabolism is found in the neocortex [26].

Here, we evaluated whether regional FDG uptake measured on PET could be used as a non-invasive tool for evaluating the status of neuroinflammation. We found that hippocampal glucose metabolism in $\mathrm{AD}$ is closely 
related to disease-associated metabolic reconfiguration of microglia and that hippocampal glucose metabolism using PET in the 5xFAD AD mouse model was correlated with microglial metabolism. Single-cell level analyses were used to support the characteristic metabolic changes in microglia of the hippocampus in AD. Furthermore, the human AD database of PET imaging and sTREM2 were analyzed to support our findings.

\section{Methods}

\section{Animals and study designs}

Male transgenic hemizygous 5xFAD mice (B6SJL-Tg (APPSwFlLon, PSEN1*M146L* L286V) 6799 Vas/ Mmjax) were obtained from Jackson Laboratory (Bar Harbor, ME, USA). In order to obtain stable results, male mice were selected and tested because there are gender differences in the pathology of $\mathrm{AD}$ mouse models. Female wild-type (WT) mice were obtained from crossbreeding a C57BL/6 female $\mathrm{x}$ an SJL male. These mice were bred, and male transgenic hemizygous and male wild-type mice were used. They were kept under standard laboratory conditions $\left(22-24{ }^{\circ} \mathrm{C}\right.$, 12 -h light and dark cycle) with free access to water and standard feed. For FDG PET studies, a total of 19 5xFAD mice were used for FDG PET scans. FDG PET images were also acquired for age-matched WT mice $(n=21)$. PET scans were performed for 4,8 , and 12 months with \pm 0.25 months.

For the ex vivo study of FDG uptake in microglia, nine 5xFAD mice and nine WT mice were sacrificed after FDG injection. The group of mice was divided according to age; 3.5, 7, and 13 months old. Single-cell RNAsequencing (scRNA-seq) studies were performed on the brains of four mice (three $5 \times$ FAD and one WT mouse). Two-, 6-, and 9-month-old 5xFAD mice and a 3-monthold WT mouse were used to obtain hippocampal tissue. The animal study was approved by the Institutional Animal Care and Use Committee at Seoul National University (SNU-181018-6).

\section{Animal PET imaging}

PET scans were performed on a dedicated small animal PET/CT scanner (eXplore VISTA, GE Healthcare, WI). All animals were fasted for at least $8 \mathrm{~h}$ before the start of the study. Animals were anesthetized with $2 \%$ isoflurane at $1 \mathrm{~L} / \mathrm{min}$ oxygen flow for 5-10 min. FDG (10.511.7 MBq) was injected by an intravenous bolus injection. Static emission scans at $45 \mathrm{~min}$ after the injection were acquired. During the FDG uptake period, animals were awake and then anesthetized $10 \mathrm{~min}$ before PET/ CT scans to reduce anesthetic effects on microglia [27]. Emission scans were acquired for $20 \mathrm{~min}$. The energy window for the scan was $400-700 \mathrm{keV}$ and images were reconstructed by a three-dimensional ordered-subsets expectation maximum (OSEM) algorithm with attenuation, random and scatter correction. The final voxel size was $0.3875 \times 0.3875 \times 0.775 \mathrm{~mm}$.

\section{Animal PET analysis}

Brain PET scans were manually examined for the quality control. All PET images were spatially normalized to the mouse brain template [28]. The spatial normalization was performed by Statistical Parametric Mapping (SPM8, University College of London, London, UK). To obtain hippocampal FDG uptake, we manually drew the right and left hippocampus on the brain template. The voxel counts were normalized to the global brain uptake in each PET image using a brain mask. Hippocampal FDG uptake was defined by using the relative value to global brain uptake.

\section{Cell FDG uptake study}

After fasting for at least $8 \mathrm{~h}, 20.3-27.4 \mathrm{MBq}$ FDG was injected into $5 \mathrm{xFAD}$ and age-matched controls intravenously. The hippocampus was isolated from the brain. Liver tissue was cut out and weighed at 40 minutes after injection. FDG uptake by the liver and microglia from the hippocampus was measured with a gamma counter (Packard Cobra II, GMI, NM, USA). Hippocampal FDG uptake values of microglia were estimated by the normalized value of counts per minute (cpm). FDG uptake values in the liver were calculated to $\mathrm{cpm} / \mathrm{mg}$. The FDG uptake values in microglia were divided by FDG uptake of the liver for each mouse. The FDG uptake values per cell were calculated using the total cell number.

\section{Cell sorting}

The microglia were isolated according to the manufacturer's instructions for magnetic-activated cell sorting (MACS) cell separation with mouse CD11b+ MicroBeads (Miltenyi Biotec, Bergisch Gladbach, Germany). The astrocytes were isolated according to the manufacturer's instructions for MACS cell separation with mouse ACSA-2 (astrocyte cell surface antigen-2) MicroBeads (Miltenyi Biotec, Bergisch Gladbach, Germany). The neurons were isolated according to the manufacturer's instructions for MACS cell separation with mouse neuron isolation kit (Miltenyi Biotec, Bergisch Gladbach, Germany).

\section{RT-PCR and quantitative PCR}

RNA was isolated from sorted brain cells with TRIzol reagent (ambion) according to the manufacturer's instructions. Reverse transcription of RNA was performed using a thermal cycler (Bio-Rad, T100). cDNA samples were amplified with primers. Then agarose gel electrophoresis was performed to analyze PCR products. For quantitative PCR, cDNA samples were mixed with SYBR 
green master mix (Takara) and loaded on an Applied Biosystems 7500. The mRNA levels of target gene, Trem2, were normalized to mRNA levels of each betaactin. Primer information: Hexb: forward 5' GCTGTTGGTGAGAGACTCTGGA3', reverse 5' GAGGTTGTGCAGCTATTCCACG3'; Snap25: forward 5'TTGGCTGAAACTATGTGAAATGGA3', 5' ATGGTGATTAACAAGAGCCAGACG3'; Slc1a3: forward 5'TGTGCTTGTTTATGTCCCTACC3', reverse 5'TCTCCTGCTGTGTTTTCTTCC3'; Trem2: forward 5'GGAACCGTCACCATCACTCT3', reverse 5' ATGCTGGCTGCAAGAAACTT3'; Tyrobp: forward 5' GATTGCCCTGGCTGTGTACT3', reverse 5' CTGGTCTCTGACCCTGAAGC3'; beta-actin: forward 5'AAGACCTCTATGCCAACACAGT3', reverse 5' GCTCAGTAACAGTCCGCCTA3'

\section{Single cell dissociation from the hippocampus}

For RNA sequencing, the Papain Dissociation System (Worthington, NJ, USA) was used to separate the hippocampus into single cells according to the manufacturer's instructions. Dead Cell Removal Kit (Miltenyi Biotec, Bergisch Gladbach, Germany) was performed before loading to $10 \times$ to remove the dead cells.

For microglial dissociation, hippocampus tissue was incubated in $1 \mathrm{mg} / \mathrm{mL}$ papain, $5 \% \mathrm{D}-(+)$-trehalose dehydrate, $0.5 \mathrm{mM}$ DL-2-amino-5-phosphonopentanoic acid, 100 units of DNase based on hibernate A without calcium and magnesium buffer in a $37^{\circ} \mathrm{C}$ shaking incubator for $1 \mathrm{~h}$. The cells were washed 3 times and then agitated using a pipette tip and passed through a $70-\mu \mathrm{m}$ filter mesh. Discontinuous density gradient buffer $(0.6$ $\mathrm{mg} / \mathrm{mL}$ ovomucoid inhibitor-albumin, 5\% D-(+)-trehalose dehydrate, $0.8 \mathrm{mM}$ DL-2-amino-5-phosphonopentanoic acid, 100 units of DNase based on hibernate A without calcium and magnesium buffer) was prepared in a tube. The cell layer suspension was carefully maintained on the gradient buffer, and then centrifuged at $900 \mathrm{rpm}$ for $6 \mathrm{~min}$ and the dissociated cell pellet was resuspended.

\section{Single cell RNA-sequencing}

The purified cells obtained from the hippocampus were sequenced using the Chromium single cell gene expression platform (10× Genomics). Approximately 10001500 cells from each dissected brain were directly loaded into each sample well following the manufacturer's instructions. All samples were combined into droplets with barcoded beads. Manufacturer specifications were followed for generation of the barcoded libraries and then the samples were sequenced to an average depth of 40,000-60,000 reads on an Illumina HiSeq 2500 sequencer.

\section{Analysis of single cell RNA-sequencing}

scRNA-seq samples were pseudoaligned to the ENSEMBL GRCm38 Mus musculus transcriptome using Kallisto, version 0.45 .0 with the default option [29]. The reads were aligned to the index using Kallisto-bus [30]. Exonic counts for each barcode were estimated after the corrected BUS file with 'bustools correct' and 'bustools sort' using specifying " $10 \times$ v2" chemistry as an option. After the estimation of gene counts using pseudoalignment, data were loaded using Seurat (version 3.0.0) [31]. All data were merged using canonical correlation analysis (CCA) as a part of the Seurat package. The cells were filtered for further analysis based on the following parameters: expression of at least 200 genes and at most 4000 genes to exclude cell duplets, and less than $10 \%$ of transcripts of mitochondrial genes. The transcript counts were $\log$ transformed with multiplication of scaling factor 10,000. Variable features (nfeatures $=2000$ ) were identified based on a variance stabilizing transformation. Next, principal component analysis (PCA) was run on variable genes, and the first ten PCs were selected for clustering analyses. Cells were clustered using the FindClusters function in Seurat with default settings, resolution $=0.2$. The marker genes of each cluster were identified by the FindAllMarkers function in Seurat. To visualize the transcript data, $\mathrm{t}$-distributed stochastic neighborhood embedding ( $\mathrm{t}-\mathrm{SNE}$ ) was used.

A subset of microglia was selected by specific clusters of microglia after the clustering of all cells. The subclusters of microglia were determined by another clustering process of the microglial subset. The highly variable genes $(n=2000)$ were reselected for the microglial subsets and then PCA was performed. The clusters of microglia were identified using the FindClusters function with resolution $=0.1$.

\section{Estimation of glucose metabolism features of cells}

The glucose metabolism features of cells were estimated by gene sets defined by Kyoto Encyclopedia of Genes and Genomes (KEGG) pathways. The specific metabolic pathways of mouse related to glucose metabolism were downloaded by GSKB for mouse [32]: 'Glycolysis and Gluconeogenesis' and 'Oxidative phosphorylation'. A gene set of glucose transporters was selected (from Glut1 to Glut12) for use as another gene set related to glucose uptake. The cellular scores of the gene sets were estimated by the AddModuleScore function in Seurat.

\section{Human FDG PET and CSF soluble TREM2 data}

In this study, human FDG PET data and CSF sTREM2 data were collected from the Alzheimer Disease Neuroimaging Initiative (ADNI) (http://adni.loni.usc.edu) database. To perform correlation analysis between FDG PET and CSF sTREM2, 98 patients: AD 27, normal controls 
(CN) 23, and mild cognitive impairment (MCI) 47, who underwent FDG PET, CSF sTREM2, and MRI were selected. The CSF sTREM2 was downloaded from the ADNI database (http://adni.loni.usc.edu). Among 47 MCI patients, 37 patients underwent more than 2 years of follow-up examinations. MCI patients were divided into two groups, $\mathrm{MCI}$ converters (MCI-C) and $\mathrm{MCI}$ non-converters (MCI-NC) according to follow-up diagnosis. Nineteen patients progressed to AD before 2 years, and they were defined as MCI-C. Eighteen patients did not progress to $\mathrm{AD}$ within 2 years and were defined as MCI-NC.

The ADNI (http://www.loni.usc.edu/) is a multicenter longitudinal study launched in 2003 as part of a $\$ 60$ million, 5-year public-private partnership led by Principal Investigator Michael W Weiner. The purpose of the ADNI has been to develop and validate biomarkers for predicting the progression of $\mathrm{MCI}$ and/or early $\mathrm{AD}$ using serial MRI, PET, other biological markers, and clinical and neuropsychological data. Many coinvestigators from a broad range of academic institutions and private corporations work to find participants, and they have recruited participants from over 50 sites across the US and Canada. The institutional review boards of all participating institutions approved imaging and laboratory studies, and all participants signed a written informed consent form. For up-to-date information, see http://www.adni-info.org.

\section{Hippocampal FDG uptake evaluation for human PET}

We evaluated FDG uptake of the hippocampus using partial volume correction. Input PET images were standardized to have the same voxel size $(1.5 \times 1.5 \times 1.5$ $\mathrm{mm})$. A partial volume correction process was performed using a PVElab software package [33]. PET images were coregistered to T1-weighted MR images. Segmentation of MR data based on MNI atlas labeling was performed and voxelwise partial volume correction was performed using a method that combines the approach of modified Müller-Gärtner proposed by Rousset et al. [34]. Count normalization was performed based on global intensity of individual.

\section{Statistical analysis}

The comparison of hippocampal FDG uptake between two groups, $5 \times \mathrm{xFD}$ and WT mice, was performed using Mann-Whitney tests. The correlation between two continuous variables was estimated by Pearson's correlation. The comparison of glucose metabolism features of each cell cluster between different mice was conducted by one-way ANOVA. The comparison of FDG uptake in the human hippocampus was performed by MannWhitney tests with Bonferonni multiple test comparison. Correlation analysis between hippocampal FDG uptake and sTREM2 levels was performed by Spearman correlation analysis.

\section{Results \\ FDG PET shows hippocampal hypermetabolism in AD mice}

To investigate the metabolic change in the hippocampus of $\mathrm{AD}$, we performed in vivo imaging using FDG PET and collected microglia to quantify FDG uptake. In addition, scRNA-seq of the hippocampus was also performed to investigate how cell-level metabolic gene signatures change in vivo (Fig. 1A). First, we compared FDG uptake in the hippocampus of 5xFAD mice and WT mice after preprocessing using a manually defined hippocampus on the normalized brain (Fig. 1B). As a result, 4-month-old mice showed no significant difference in hippocampal FDG uptake. Eight-month-old and 12month-old 5xFAD mice showed significantly higher FDG uptake in the hippocampus than WT mice $(p=$ 0.016 and $p=0.032$, 8-month-old; $p=0.009$ and $p=$ $0.06,12$-month-old; right and left hippocampus, respectively) (Fig. 1C, Supplemental figure 1).

We then hypothesized that hippocampal hypermetabolism was associated with the accumulation of inflammatory cells. Ex vivo FDG uptake in microglia in the hippocampus was examined to reveal whether hippocampal hypermetabolism depended on metabolic changes in microglia or non-microglial cells. After FDG injection, microglia in the hippocampus were selectively collected to measure radioactivity. As a result, at 7.5 months of age, 5xFAD mice showed significantly higher FDG uptake in microglia than WT mice. At 3.5 and 13 months of age, 5xFAD mice also showed a trend of higher FDG uptake in microglia than WT mice. Other non-microglial cells showed no significant difference in FDG uptake regardless of age (Fig. 1D, E). We also acquired FDG uptake in subtypes of non-microglial cells, including astrocytes and neurons in 6-month-old 5xFAD. FDG uptake in microglia was higher in 5xFAD, while astrocytes and neurons showed no significant difference in FDG uptake between 5xFAD and WT mice (Supplementary figure 1B, C).

\section{Single-cell analysis reveals changes in glucose metabolic signatures of microglia of $A D$ mice}

As hippocampal hypermetabolism in $5 \mathrm{xFAD}$ was associated with increased uptake in the microglia of the hippocampus, we investigated the metabolic signatures of various cell types of the hippocampus at the single-cell level. The scRNA-seq data were obtained from the hippocampus of $5 \mathrm{xFAD}$ mice of different ages $(2,6$, and 9 months old) and a WT mouse. A total of 5812 cells (1898, 1200, and 933 cells for 2-, 6-, and 9-month-old 5xFAD and 1781 cells for WT) were clustered and 


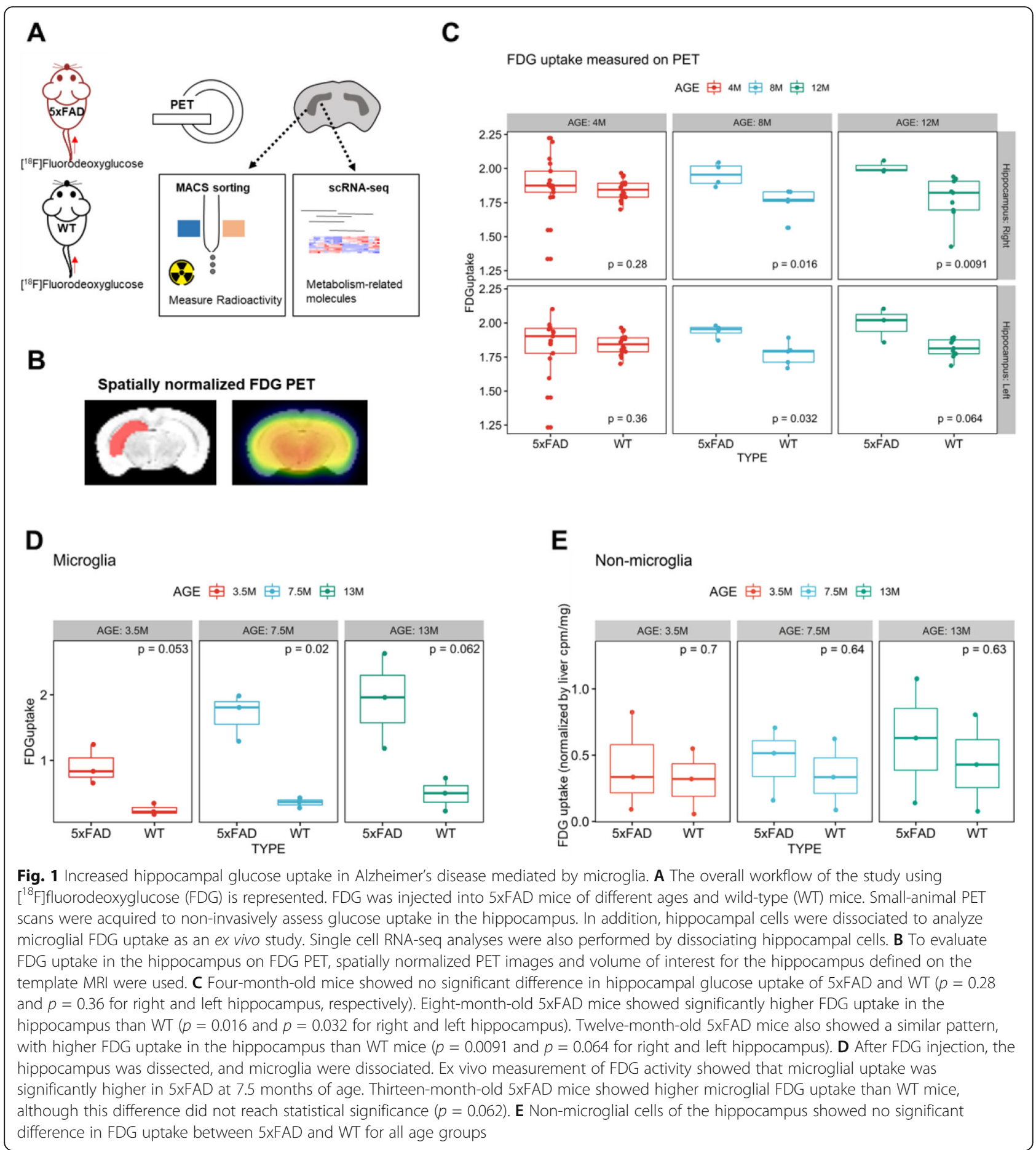

analyzed. Accordingly, nine different cell clusters were identified (Fig. 2A, B). The distribution of cell types was changed according to the aging of $5 x F A D$ mice (Supplemental figure 2). The identified markers and alleged cell type-specific gene expression of each cluster are represented in Supplemental figure 3 and Supplemental figure 4. To compare the metabolic properties of each cell type, the enrichment scores of glycolysis, OXPHOS, and glucose transporters (GLUTs) were estimated. The enrichment scores of gene sets were evaluated for each cell. These glucose metabolism features were significantly different among 5xFAD mice of different ages and WT in specific clusters (Fig. 2C, Supplemental figure 5). In particular, glycolysis, OXPHOS, and GLUTs of different mice were significantly different in the microglia 1 cluster. For the microglia 1 cluster, the enrichment scores of 


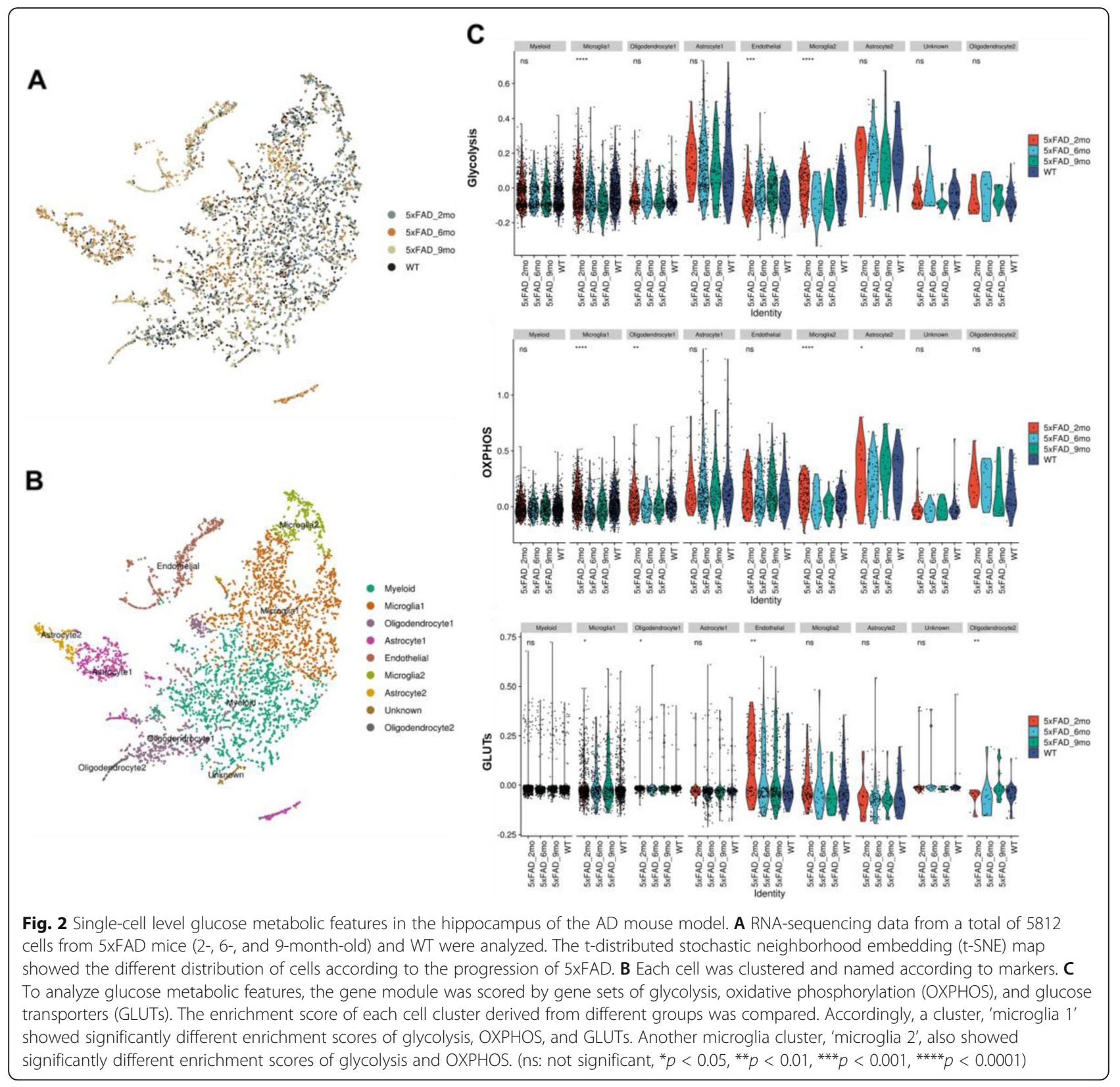

glycolysis and OXPHOS were lower and the score of GLUTs was higher in the 9-month-old 5xFAD mouse. Another microglia cluster, the microglia 2 cluster, also showed different glycolysis and OXPHOS scores between different 5xFAD mice. The enrichment scores of glycolysis and OXPHOS of the microglia 2 cluster were lower in the 9-month-old 5xFAD mouse. Other clusters, including astrocytes and oligodendrocytes, did not show different glucose metabolic features among the different mice. Thus, we hypothesized that highly changed glucose metabolic signatures in microglia affected hippocampal glucose hypermetabolism.

\section{Reprogrammed glucose metabolism of microglial} subtypes characterized by increased GLUT and decreased glycolysis and OXPHOS

As glucose metabolic features of microglia were significantly different between mice, microglial subsets were further investigated. Four different subtypes of microglia were identified (Fig. 3A). The markers of different microglial subtypes are represented in Supplemental figure 6. The proportion of subtypes of microglia changed according to the age of $5 \mathrm{xFAD}$ mice (Fig. 3B, C). In particular, subtype 1 and 3 were remarkably decreased in 6-month-old and 9month-old 5xFAD mice. Subtype 2 was relatively increased in 6-month-old and 9-month-old 5xFAD mice. 


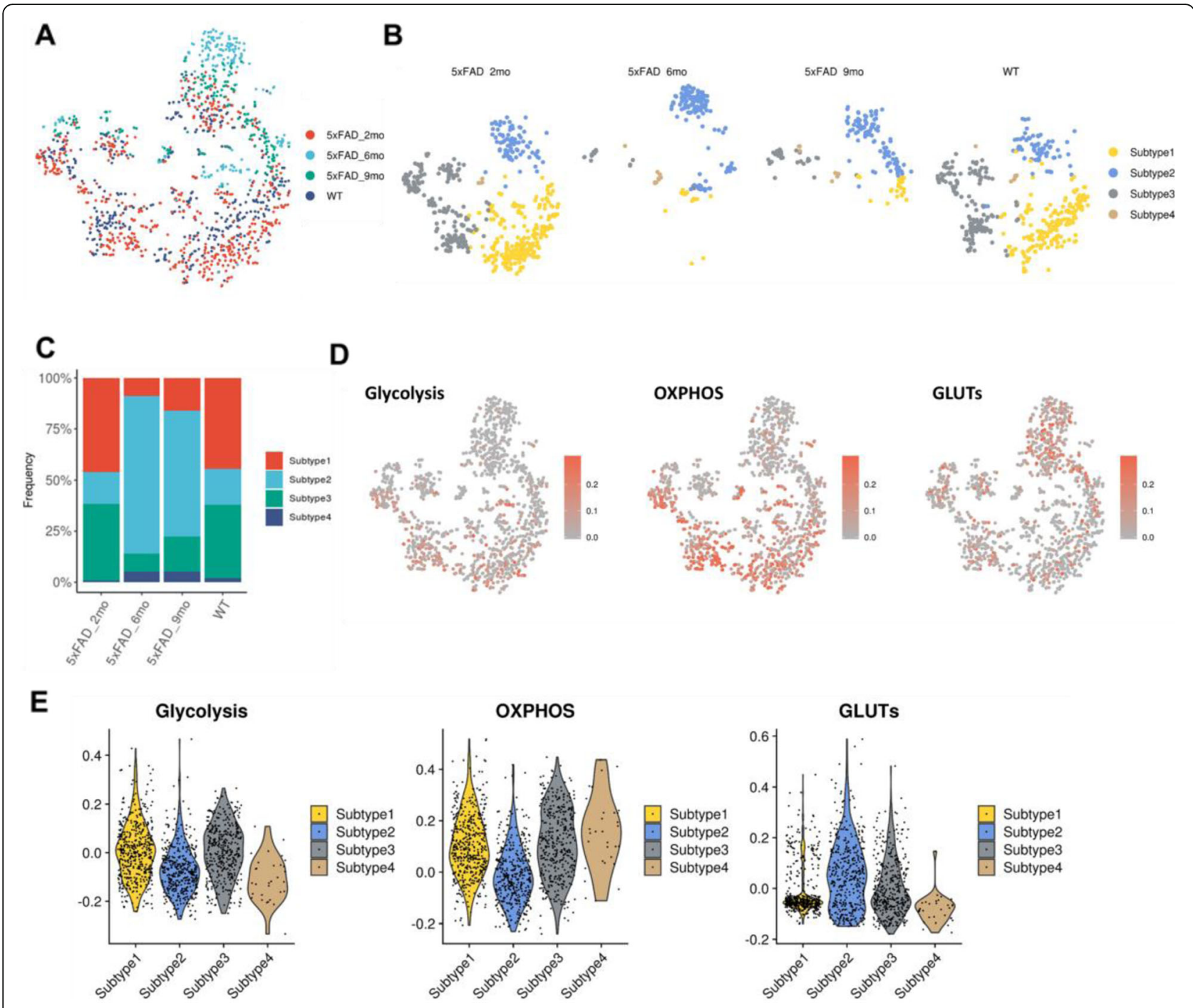

Fig. 3 Reconstitution of glucose metabolism features of hippocampal microglia in the AD mouse model. A The t-SNE map represents the hippocampal microglia of different mouse groups. B Four different subtypes of hippocampal microglia were identified. The distribution pattern of microglial subtypes was changed according to mouse groups. $\mathbf{C}$ The proportion of subtypes of hippocampal microglia changed according to the age of 5xFAD mice. The 6-month-old and 9-month-old mice showed relatively more hippocampal microglia subtype 2. D The glucose metabolic features of microglial subtypes are represented. The enrichment score of GLUTS showed a different distribution pattern than glycolysis and OXPHOS enrichment scores. E Glucose metabolism features of the hippocampal microglial subtypes were different. Glycolysis and OXPHOS enrichment scores were lower in 'Subtype 2', while GLUT enrichment score was higher in 'Subtype 2' than other subtypes

The glucose metabolism features of microglial subtypes are represented (Fig. 3D). The enrichment scores of glycolysis and OXPHOS were relatively high in the subtype 1 and 3 microglia (Fig. 3E). On the other hand, subtype 2 microglia showed relatively low glycolysis and OXPHOS profiles and a high number of GLUTs. These findings showed that the progression of $5 x F A D$ pathology was associated with the increased microglial subtypes with high GLUTs and decreased microglial subtypes with high OXPHOS and glycolysis.
Hippocampal glucose metabolism as a surrogate of microglia-mediated inflammation in the human brain Since the mouse FDG PET study and scRNA-seq analyses suggested that microglial reconfiguration underlies hippocampal hypermetabolism, we explored whether the hippocampal metabolism evaluated in human imaging could be a surrogate of metabolic changes in microglia as an $\mathrm{AD}$ pathology (Fig. 4A). We used the human data of sTREM2 measured in CSF as surrogates of microglial inflammation. The sTREM2 level was not different among the three diagnostic groups, $\mathrm{AD}, \mathrm{MCI}$, and $\mathrm{CN}$ 


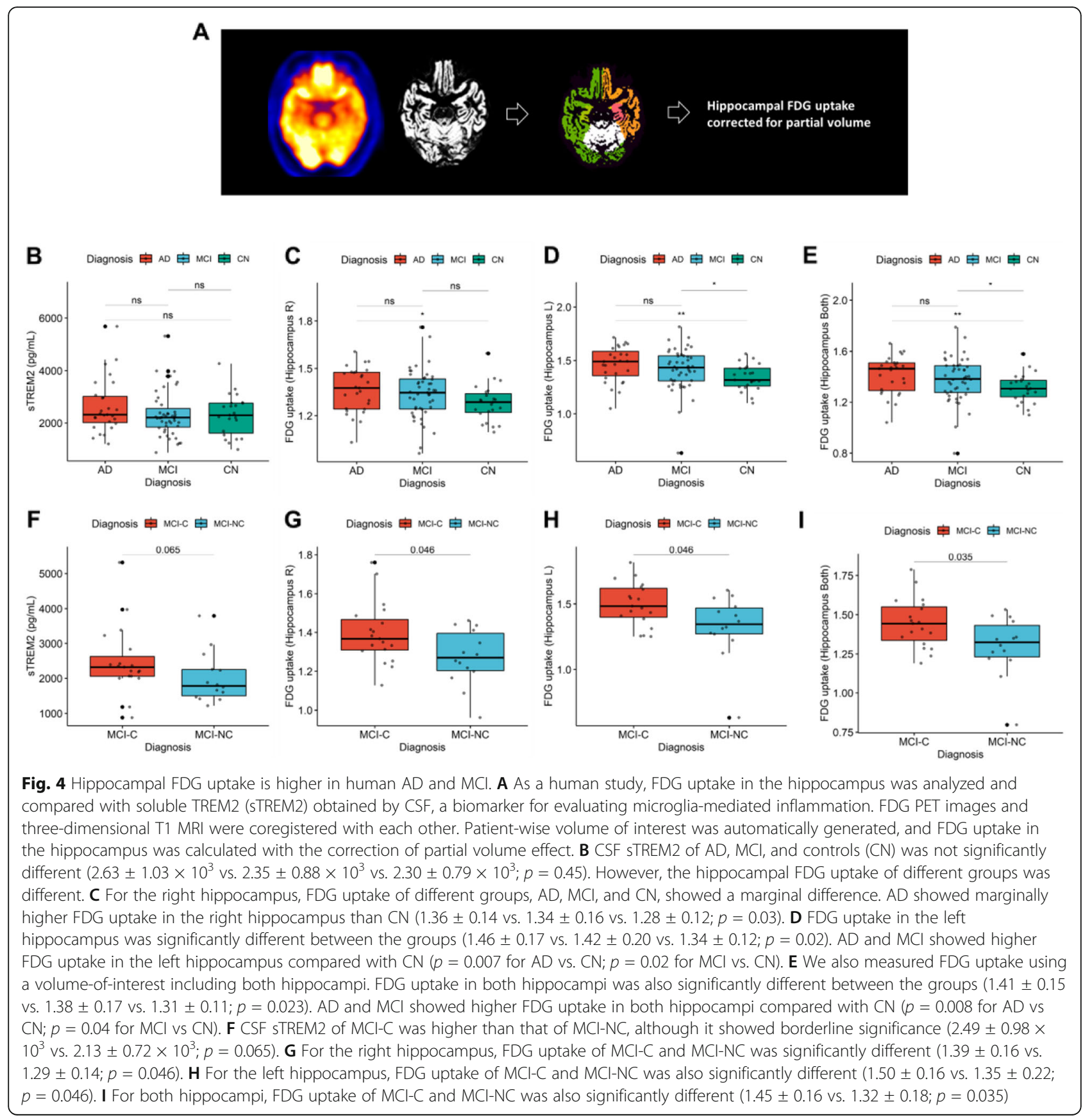

(Fig. 4B). The hippocampal FDG uptake was estimated by using a partial volume correction method based on structural MR images [35]. Accordingly, hippocampal FDG uptake was significantly increased in the $\mathrm{AD}$ brain compared with the $\mathrm{CN}$ brain (Fig. 4C-E). We compared hippocampal FDG uptake of patients with MCI-C and MCI-NC to investigate whether it could be a predictive marker for the progression of MCI. The sTREM2 of MCI-C was higher than that of MCI-NC, although it showed borderline statistical significance (Fig. 4F). FDG uptake was significantly higher in MCI$\mathrm{C}$ than MCI-NC in right and left hippocampus (Fig. 4G-I).
sTREM2 showed a trend of positive correlation with hippocampal FDG uptake $(r=0.23, p=0.025$ for left hippocampal FDG uptake; $r=0.14, p=0.19$ for right hippocampal FDG uptake; $r=0.18, p=0.081$ for both hippocampi; Fig. 5A-C). These positive associations were more prominent in the subgroup of MCI subjects (Fig. 5D-F). The association between sTREM2 and hippocampal FDG uptake was not found in $\mathrm{AD}$ and $\mathrm{CN}$ (Supplemental figure 7). However, hippocampal FDG uptake of $\mathrm{AD}, \mathrm{MCI}$, and $\mathrm{CN}$ was not associated with the severity of a cognitive score, Alzheimer's disease 


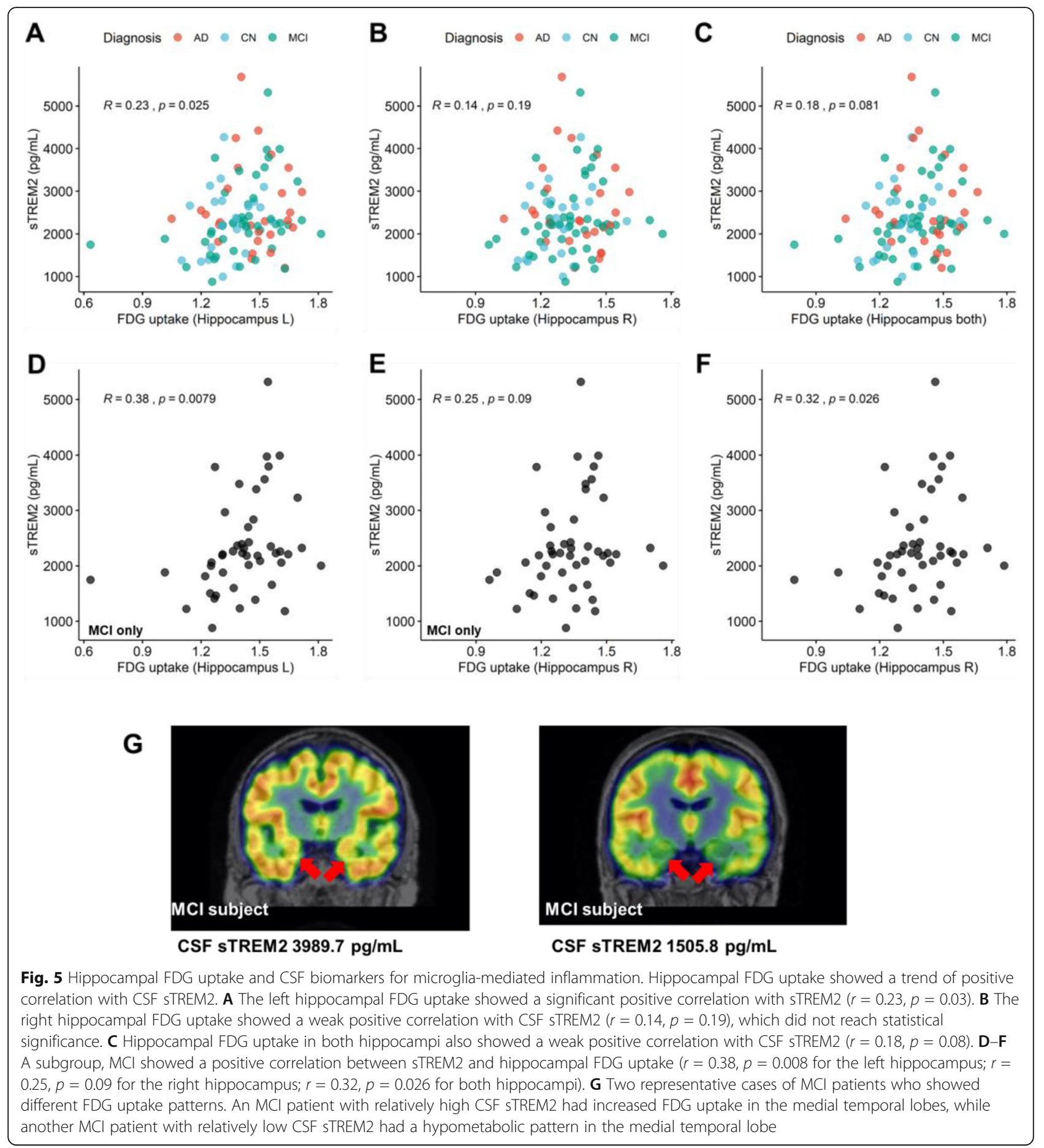

assessment scale (ADAS13) (Supplementary figure 8). sTREM2 was also not correlated with ADAS13 in all subgroups (AD, $\mathrm{MCI}$ and $\mathrm{CN}$ ). The representative cases of FDG PET images of MCI subjects with different sTREM2 levels are represented in Fig. 5E. Relatively high FDG uptake in medial temporal cortices was found in a subject with higher sTREM2 levels.

\section{Discussion}

The evaluation of neuroinflammation in AD become an important pathophysiologic biomarker as it has been demonstrated to be a predictor of cognitive decline in patients using CSF inflammation markers as well as animal models [36, 37]. As a key player in neuroinflammation, it is necessary to understand the characteristics of microglia in AD brain and observe changes of microglial 
activation to predict progression and cognitive changes in $\mathrm{AD}$ continuum.

One of the key changes in microglia-mediated inflammation is metabolism, as immune cells depend on various metabolic pathways according to energy demands. The activation of the innate immune system is associated with a metabolic shift from oxidative phosphorylation (OXPHOS) to glycolysis, which is a rapid process of energy production [8]. These active metabolic processes can be suppressed by chronic inflammatory processes due to broad defects in energy metabolism that underlie immunoparalysis [13]. This close link between metabolism and immune cells is also found in AD. TREM2, a key surface receptor for the microglial response to neurodegeneration-related proteins, was associated with the metabolic fitness of microglia to enhance glycolysis to protect them from neurodegenerative proteins. According to a previous study, TREM2-deficiency induces impaired energy metabolism in microglia associated with defective mTOR pathway [14]. Furthermore, a recent study showed that amyloid beta exposure to microglia induced microglial reprogramming from OXPHOS to glycolysis at the acute phase; however, both were reduced after chronic exposure similar to the process of immunoparalysis [15]. As microglia with proinflammatory response, so-called M1phenotypes in macrophages, are associated with increased glycolysis [38], this energy defect implies immune tolerance mediated by $\mathrm{AD}$ pathology in the hippocampus. Notably, while both glycolysis and OXPHOS were decreased, glucose uptake of hippocampal microglia was increased in our study. This increased glucose uptake was identified in ex vivo microglia as well as in vivo PET imaging. Moreover, a subset of microglia with high GLUTs was increased in 5xFAD mice. As increased GLUT1 is similar with the common pathway of stress condition even in broad defects of energy metabolism [39], the finding of increased GLUT1 and FDG uptake in hippocampal microglia could be associated with metabolic reprogramming of chronic inflammatory changes resulting from AD pathology. The specific metabolic pathways of microglia with immunoparalysis are unknown, and possibly, increased storage pathways during both decreased glycolysis and OXPHOS. As future work, metabolic pathways according to microglial activation status should be studied to fully understand the metabolic pathway of glucose of microglia with increased GLUT1 with immunoparalysis. Furthermore, scRNA-seq data were acquired from limited mice as well as limited brain regions. Considering the spatial diversity in microglia [40] and different metabolic patterns of hippocampus on PET according to our study, further detailed scRNA-seq analyses should be performed to understand glucose metabolism of various immune cell types in the brain.
Recent progress has been made in image-based phenotyping of $\mathrm{AD}$, and the changes in key biomarkers are spatially and temporally different according to disease progression [41, 42]. For example, volume loss evaluated by MRI is typically found in the hippocampus, while reduced glucose metabolism is mainly found in the posterior cingulate, lateral temporal, superior parietal, and medial frontal cortices [42]. The reduced glucose metabolism was spatially associated with amyloid deposits and regarded as decreased neuronal activity resulting from AD pathology [43]. While the precuneus and posterior cingulate cortex are the common brain regions that show both hypometabolism and volume loss according to the progression of AD [41], FDG uptake patterns in the hippocampus are equivocal. Despite the consistent association of hippocampal atrophy and cognitive decline, FDG uptake in the hippocampus is relatively preserved and sometimes increased [22, 23, 44]. The animal $\mathrm{AD}$ model has also showed equivocal results. A recent study presented regional FDG uptake in 12-month-old 5XFAD mice using autoradiography as well as PET, while it showed no definite difference in FDG uptake compared with wild types [45]. In spite of different protocols and experimental settings from our animal PET studies, this study showed insignificantly higher FDG uptake in the female 5XFAD mice hippocampus than female wild-type mice [45]. The results of increased FDG uptake among these studies were associated with partial volume correction as hippocampal atrophy could cause underestimate the measurement of FDG uptake on PET. According to our findings, one of the reasons for relatively preserved glucose uptake was microglial reconfiguration in the hippocampus. Furthermore, the microgliamediated inflammatory response alters adult hippocampal neurogenesis [46], which may also affect FDG uptake in the hippocampus. Notably, as FDG uptake usually depends on neuronal activity, hippocampal FDG uptake patterns on PET could depend on multiple cell types. Accordingly, glucose uptake in the hippocampus noninvasively measured by FDG PET could be a biomarker associated with microglia-mediated inflammation. Notably, microglia-mediated inflammation is increased at the early stage of $\mathrm{AD}$ and reduced at the late stage according to CSF inflammatory markers [47, 48]. In this regard, FDG uptake in the hippocampus reflecting microglial reconstitution could be varied in $A D$, increased at the early stage and reduced at the late stage. Further study is needed to assess hippocampal FDG uptake according to disease progression.

Recently, the role of microglia in AD pathophysiology has received considerable attention. In particular, DAM were identified in neurodegenerative disorders and regarded as a potential target of treatment [49]. The key hypothesis is the protective role of DAM in the immune 
response to neural tissue damage, such as innate immune response to damage-associated molecular patterns [5]. Thus, in terms of clinically applicable biomarker development, a subset of microglia that reflects the current status of neuroinflammation caused by AD pathophysiology is needed because microglia are highly heterogeneous and dynamically changed. To date, TSPO PET has been widely studied in AD to non-invasively evaluate microglial inflammation. However, TSPO-positive microglia are different from DAM activated by the TREM2 pathway $[50,51]$. In this regard, it is difficult to reflect complex and dynamic changes in microglial subsets in neurodegenerative disorders. Another emerging biomarker for assessing DAM is CSF sTREM2, which can induce DAM to protect against amyloid beta-induced neuronal damage $[52,53]$. According to previous studies, sTREM2 can reflect disease progression in terms of microglia-mediated inflammation and be a potential biomarker of AD [54]. Despite the emerging role of CSF sTREM2 in reflecting microglia-mediated inflammation, one of the limitations considering clinical usage is its invasiveness. As we found that sTREM2 in CSF was positively correlated with hippocampal glucose uptake noninvasively measured by FDG PET, it could be a clinically feasible surrogate to assess microglia-mediated inflammation. Furthermore, FDG PET is a common and widely used imaging modality for study of $\mathrm{AD}$; thus, it is relatively easy to use as a surrogate biomarker. Interestingly, sTREM2 was particularly correlated with hippocampal FDG uptake in MCI patients among whom converters showed higher uptake than non-converters. Furthermore, the correlation between hippocampal FDG uptake and sTREM2 was mainly found in MCI, while it was not correlated in $\mathrm{AD}$ and $\mathrm{CN}$. It supports microglia-mediated inflammation during the disease course as STREM2 is elevated at the early stage of AD and decreased at the late stage of AD [47, 55]. Hippocampal FDG uptake measured by $\mathrm{PET}$ in late stage $\mathrm{AD}$ or normal aged subjects may mainly depend on non-microglial cells due to relatively low glucose uptake in microglia. This might be a cause of the lack of correlation between sTREM2 and hippocampal FDG uptake in AD patients and normal controls. Thus, it does not reflect microglial metabolic status related to the sTREM2 pathway for normal subjects or late stage AD. Therefore, hippocampal FDG uptake, as well as sTREM2, could be a good biomarker of MCI or early AD for predicting outcome. Additional validation studies for investigating FDG uptake in the hippocampus according to the course of $\mathrm{AD}$ and the microglial activation pathway will test this hypothesis.

\section{Conclusions}

We found that increased glucose uptake in the hippocampus was associated with microglial reconfiguration.
At the single-cell level, a subset of hippocampal microglia that showed high GLUTs and low glycolysis and OXPHOS was associated with the progression of the $\mathrm{AD}$ mouse model. This microglia-mediated glucose uptake in the hippocampus could be estimated by FDG PET, one of the most widely used non-invasive imaging modality. This association between microglia-mediated inflammation and FDG uptake in the hippocampus was recapitulated in human imaging by the positive correlation of CSF sTREM2 and hippocampal FDG uptake. Microglia-mediated inflammation in the brain has been regarded as a key pathophysiology of AD. Nonetheless, because of complicated dynamic changes in microglia affecting both the protection and progression of AD pathology represented by amyloid beta, clinical translation of these biological changes of dysfunctional microglia has been limited. Our findings will provide a clinically feasible surrogate marker to monitor the status of microgliamediated inflammation by using widely used PET imaging. Furthermore, the characteristic microglial metabolism of AD analyzed by integrating imaging, scRNAseq, and human biomarkers provides insight into new therapeutic targets of $\mathrm{AD}$ pathophysiology.

\section{Abbreviations \\ AD: Alzheimer's disease; DAM: Disease-associated microglia; PET: Positron emission tomography; TSPO: Translocator protein-18 kDa; FDG: Fluorodeoxyglucose; WT: Wild-type; scRNA-seq: Single-cell RNA- sequencing; OSEM: Ordered-subsets expectation maximum; MACS: Magnetic- activated cell sorting; CCA: Canonical correlation analysis; t-SNE: t-distributed stochastic neighborhood embedding; PCA: Principal component analysis; KEGG: Kyoto encyclopedia of genes and genomes; sTREM2: Soluble TREM2; ADNI: Alzheimer disease neuroimaging initiative; CN: Normal controls; $\mathrm{MCl}$ : Mild cognitive impairment; MCl-C: MCl converters; MCl-NC: MCI non- converters; OXPHOS: Oxidative phosphorylation; GLUT: Glucose transporter}

\section{Supplementary Information}

The online version contains supplementary material available at https://doi. org/10.1186/s12974-021-02244-6.

\footnotetext{
Additional file 1: Supplemental figure 1. FDG PET images of mice with different ages and cellular FDG uptake. (A) FDG PET images averaged across each group were represented. Note that the hippocampal FDG uptake was estimated by pre-defined volume-ofinterests after the spatial normalization of FDG PET. The hippocampal FDG uptake was increased in 8-month-old and 12-month-old 5xFAD mice compared with wild type mice with same age. (B) To know FDG uptake difference in subtypes of nonmicroglial cells, we additionally performed FDG uptake tests after cellular sorting including astrocytes and neurons in 6-month-old 5xFAD mice and WT. FDG uptake of astrocytes and neurons was not significantly different between $5 x F A D$ and WT $(*: p<0.05)$. (C) Purity of cell fractions for ex vivo FDG studies was determined by PCR. The microglia-rich fraction was identified as Hexb, the astrocyte-rich fraction as S/c1a3, and the neuron-rich fraction as Snap25. Cortical lysates were used as positive controls. Supplemental figure $\mathbf{2}$. The change in cellular landscape of hippocampus according to aging of 5xFAD mice. tSNE maps representing single cell-level transcripts were drawn for 5xFAD and wild type mice with different ages. Supplemental figure 3. Markers of hippocampal cell cluster. Cells were clustered according to the single cell-level transcripts and markers were identified. 9 different cell types were clustered and names were defined by alleged markers. The average expression value of cells of each cluster was represented. Supplemental
} 
figure 4. Expression of cell-type specific genes. Gene expression levels of cell-type specific genes were represented with t-SNE maps. Supplemental figure 5. The enrichment score of glucose metabolism-related molecules for hippocampal cells. t-SNE maps were drawn with the enrichment score of glucose metabolism modules. The enrichment score of glycolysis, oxidative phosphorylation (OXPHOS), and glucose transporters (GLUTs) was estimated by using KEGG pathway. The enrichment score was represented according to colormaps. Notably, astrocyte shows relatively higher glycolysis. GLUTs were highly expressed in endothelial cells and some types of microglial clusters. Supplemental figure 6 . Markers of microglial subtypes. Microglial subsets were further analyzed to investigate reprogrammed microglia according to the AD progression particularly in terms of the glucose metabolic profiles. Microglia were clustered into 4 subtypes by gene expression data and markers were identified. Supplemental figure 7. The correlation between hippocampal FDG uptake and CSF sTREM2 in AD and controls. The hippocampal FDG uptake was positively correlated with CSF STREM2 particularly in MCl patients, while the association was neither found in AD nor controls. FDG uptake in the left hippocampus (A) and the right hippocampus (B) was not significantly correlated with sTREM2 for AD patients, while the correlation was negative. Additionally, FDG uptake in the left hippocampus (C) and the right hippocampus (D) was not significantly correlated sTREM2 for controls. However, the correlation was positive though it did not reach a statistical significance. Supplemental figure $\mathbf{8}$. The correlation between the cognitive score and hippocampal FDG uptake. In the subgroups according to the diagnosis ( $\mathrm{CN}, \mathrm{MCl}$ and $\mathrm{AD}$ ), hippocampal FDG uptake was not associated with the severity of a cognitive score, Alzheimer's disease assessment scale (ADAS13) (above). In addition, sTREM2 was also not correlated with ADAS13 in all subgroup (AD, $\mathrm{MCl}$ and $\mathrm{CN}$ ) (below).

\section{Acknowledgements}

Data collection and sharing for this project was funded by the Alzheimer's Disease Neuroimaging Initiative (ADNI) (National Institutes of Health Grant U01 AG024904) and DOD ADNI (Department of Defense award number W81XWH-12-2-0012). ADNI is funded by the National Institute on Aging, the National Institute of Biomedical Imaging and Bioengineering, and through generous contributions from the following: AbbVie, Alzheimer's Association; Alzheimer's Drug Discovery Foundation; Araclon Biotech; BioClinica, Inc.; Biogen; Bristol-Myers Squibb Company; CereSpir, Inc;; Eisai Inc; Elan Pharmaceuticals, Inc.; Eli Lilly and Company; Eurolmmun; F. Hoffmann-La Roche Ltd and its affiliated company Genentech, Inc.; Fujirebio; GE Healthcare; IXICO Ltd.; Janssen Alzheimer Immunotherapy Research \& Development, LLC.; Johnson \& Johnson Pharmaceutical Research \& Development LLC: Lumosity; Lundbeck; Merck \& Co., Inc.; Meso Scale Diagnostics, LLC.; NeuroRx Research; Neurotrack Technologies; Novartis Pharmaceuticals Corporation; Pfizer Inc:; Piramal Imaging; Servier; Takeda Pharmaceutical Company; and Transition Therapeutics. The Canadian Institutes of Health Research is providing funds to support ADNI clinical sites in Canada. Private sector contributions are facilitated by the Foundation for the National Institutes of Health (www.fnih.org). The grantee organization is the Northern California Institute for Research and Education, and the study is coordinated by the Alzheimer's Disease Cooperative Study at the University of California, San Diego. ADNI data are disseminated by the Laboratory for Neuro Imaging at the University of Southern California.

\section{Authors' Contributions}

H.C. and D.S.L. designed the study. Y.C., Y.L., H.K., and S.K. performed animal imaging study. Y.C. and H.K. performed ex vivo analysis and single-cell experiments. H.C. and Y.C. analyzed animal imaging and single cell RNAsequencing data. H.C. analyzed human imaging studies. D.W.H. and D.S.L. critically contributed to the interpretation of results. All authors wrote the paper. The author(s) read and approved the final manuscript.

\section{Funding}

This research was supported by the National Research Foundation of Korea Grant funded by the Korea Government (Nos. NRF-2019K1A3A1A14065446, NRF-2019R1F1A1061412, and NRF-2020M3A9B6038086, NRF-

2017M3C7A1048079, NRF-2020R1A2C2101069) and also supported by a grant of the Korea Health Technology R\&D Project through the Korea Health
Industry Development Institute (KHIDshow [?A3B2 h=0pt,128?]), funded by the Ministry of Health \& Welfare, Republic of Korea (H119C0339).

\section{Availability of data and materials}

Single cell RNA-sequencing data are available at Gene Expression Omnibus (GSE150934). Human brain imaging data and clinical features are available in ADNI database (http://adni.loni.usc.edu/)

\section{Declarations}

\section{Ethics approval and consent to participate}

The animal study was approved by the Institutional Animal Care and Use Committee at Seoul National University (SNU-181018-6). Patient data we used were acquired by a publicly available dataset that removed patient identifiers. The publicly available data were collected with patients' informed consent approved by the institutional review boards of all participating institutions following 1964 Helsinki Declaration and its later amendments or comparable ethical standards.

\section{Consent for publication}

Not applicable

\section{Competing interests}

The authors declare that they have no competing interests.

\section{Author details}

'Department of Nuclear Medicine, Seoul National University Hospital, 101 Daehak-ro, Jongno-Gu, Seoul 03080 Seoul, Republic of Korea. ${ }^{2}$ Department of Nuclear Medicine, Seoul National University College of Medicine, 101 Daehak-ro, Seoul, Jongo-Gu 03080, Republic of Korea. ${ }^{3}$ Department of Molecular Medicine and Biopharmaceutical Sciences, Graduate School of Convergence Science and Technology, Seoul National University, 101 Daehak-ro, Jongno-Gu, Seoul 03080 Seoul, Republic of Korea.

Received: 1 April 2021 Accepted: 18 August 2021 Published online: 31 August 2021

\section{References}

1. Ittner LM, Götz J. Amyloid- $\beta$ and tau - a toxic pas de deux in Alzheimer's disease. Nat Rev Neurosci. 2011;12(2):67-72. https://doi.org/10.1038/nrn2967.

2. Long JM, Holtzman DM. Alzheimer disease: an update on pathobiology and treatment strategies. Cell. 2019;179(2):312-39. https://doi.org/10.1016/j.cell.2 019.09.001.

3. Jack CR Jr, Bennett DA, Blennow K, Carrillo MC, Dunn B, Haeberlein SB, et al. NIA-AA research framework: toward a biological definition of Alzheimer's disease. Alzheimers Dement. 2018;14(4):535-62. https://doi.org/10.1016/j.ja Iz.2018.02.018.

4. Salter MW, Stevens B. Microglia emerge as central players in brain disease. Nat Med. 2017;23(9):1018-27. https://doi.org/10.1038/nm.4397.

5. Deczkowska A, Keren-Shaul H, Weiner A, Colonna M, Schwartz M, Amit I. Disease-associated microglia: a universal immune sensor of neurodegeneration. Cell. 2018;173(5):1073-81. https://doi.org/10.1016/j.cell.2 018.05.003.

6. Keren-Shaul H, Spinrad A, Weiner A, Matcovitch-Natan O, Dvir-Szternfeld R, Ulland TK, et al. A unique microglia type associated with restricting development of Alzheimer's disease. Cell. 2017:169:1276-90 e1217.

7. Mathys H, Adaikkan C, Gao F, Young JZ, Manet E, Hemberg M, et al. Temporal tracking of microglia activation in neurodegeneration at singlecell resolution. Cell Rep. 2017;21(2):366-80. https://doi.org/10.1016/j.celrep.2 017.09.039.

8. Lauro C, Limatola C. Metabolic reprograming of microglia in the regulation of the innate inflammatory response. Front Immunol. 2020;11:493. https:// doi.org/10.3389/fimmu.2020.00493

9. Sarlus H, Heneka MT. Microglia in Alzheimer's disease. J Clin Invest. 2017; 127(9):3240-9. https://doi.org/10.1172/JCl90606.

10. Ulland TK, Colonna M. TREM2-a key player in microglial biology and Alzheimer disease. Nat Rev Neurol. 2018;14(11):667-75. https://doi.org/10.1 038/s41582-018-0072-1.

11. Hansen DV, Hanson JE, Sheng M. Microglia in Alzheimer's disease. J Cell Biol. 2018;217(2):459-72. https://doi.org/10.1083/jcb.201709069. 
12. Heslegrave A, Heywood W, Paterson R, Magdalinou N, Svensson J, Johansson P, et al. Increased cerebrospinal fluid soluble TREM2 concentration in Alzheimer's disease. Mol Neurodegeneration. 2016;11:1-7.

13. Cheng S-C, Scicluna BP, Arts RJ, Gresnigt MS, Lachmandas E, GiamarellosBourboulis EJ, et al. Broad defects in the energy metabolism of leukocytes underlie immunoparalysis in sepsis. Nat Immunol. 2016;17(4):406-13. https:// doi.org/10.1038/ni.3398.

14. Ulland TK, Song WM, Huang SC-C, Ulrich JD, Sergushichev A, Beatty WL, et al. TREM2 maintains microglial metabolic fitness in Alzheimer's disease. Cell. 2017;170:649-63 e613.

15. Baik SH, Kang S, Lee W, Choi H, Chung S, Kim J-I, et al. A breakdown in metabolic reprogramming causes microglia dysfunction in Alzheimer's disease. Cell Metab. 2019;30:493-507 e496.

16. Edison P, Archer HA, Gerhard A, Hinz R, Pavese N, Turkheimer FE, et al. Microglia, amyloid, and cognition in Alzheimer's disease: An [11C](R) PK11195-PET and [11C] PIB-PET study. Neurobiol Dis. 2008;32(3):412-9. https://doi.org/10.1016/j.nbd.2008.08.001.

17. Fan Z, Brooks DJ, Okello A, Edison P. An early and late peak in microglial activation in Alzheimer's disease trajectory. Brain. 2017;140(3):792-803. https://doi.org/10.1093/brain/aww349.

18. Minoshima S, Foster NL, Sima AA, Frey KA, Albin RL, Kuhl DE. Alzheimer's disease versus dementia with Lewy bodies: cerebral metabolic distinction with autopsy confirmation. Ann Neurol. 2001;50(3):358-65. https://doi.org/1 0.1002/ana.1133.

19. Bohnen NI, Djang DS, Herholz K, Anzai Y, Minoshima S. Effectiveness and safety of 18F-FDG PET in the evaluation of dementia: a review of the recent literature. J Nucl Med. 2012;53(1):59-71. https://doi.org/10.2967/jnumed.111. 096578.

20. Patel AB, Lai JC, Chowdhury GM, Hyder F, Rothman DL, Shulman RG, et al. Direct evidence for activity-dependent glucose phosphorylation in neurons with implications for the astrocyte-to-neuron lactate shuttle. Proc Natl Acad Sci. 2014;111(14):5385-90. https://doi.org/10.1073/pnas.14 03576111

21. Zimmer ER, Parent MJ, Souza DG, Leuzy A, Lecrux C, Kim H-I, et al. [18 F] FDG PET signal is driven by astroglial glutamate transport. Nat Neurosci. 2017;20(3):393-5. https://doi.org/10.1038/nn.4492.

22. Apostolova I, Lange C, Mäurer A, Suppa P, Spies L, Grothe MJ, et al. Hypermetabolism in the hippocampal formation of cognitively impaired patients indicates detrimental maladaptation. Neurobiol Aging. 2018;65: 41-50. https://doi.org/10.1016/j.neurobiolaging.2018.01.002.

23. Ashraf A, Fan Z, Brooks D, Edison P. Cortical hypermetabolism in MCl subjects: a compensatory mechanism? Eur J Nucl Med Mol Imaging. 2015: 42(3):447-58. https://doi.org/10.1007/s00259-014-2919-z.

24. Rojas S, Herance JR, Gispert JD, Abad S, Torrent É, Jiménez X, et al. In vivo evaluation of amyloid deposition and brain glucose metabolism of 5XFAD mice using positron emission tomography. Neurobiol Aging. 2013;34(7): 1790-8. https://doi.org/10.1016/j.neurobiolaging.2012.12.027.

25. Luo F, Rustay NR, Ebert U, Hradil VP, Cole TB, Llano DA, et al. Characterization of 7-and 19-month-old Tg2576 mice using multimodal in vivo imaging: limitations as a translatable model of Alzheimer's disease. Neurobiol Aging. 2012;33(5):933-44. https://doi.org/10.1016/j.neurobiola ging.2010.08.005.

26. Bailly M, Destrieux C, Hommet C, Mondon K, Cottier J-P, Beaufils E, et al. Precuneus and cingulate cortex atrophy and hypometabolism in patients with Alzheimer's disease and mild cognitive impairment: MRI and 18F-FDG PET quantitative analysis using FreeSurfer. BioMed Res Int. 2015;2015:1-8. https://doi.org/10.1155/2015/583931.

27. Umpierre AD, Bystrom LL, Ying Y, Liu YU, Worrell G, Wu L-J. Microglial calcium signaling is attuned to neuronal activity in awake mice. Elife. 2020;9: e56502. https://doi.org/10.7554/eLife.56502.

28. Mirrione MM, Schiffer WK, Fowler JS, Alexoff DL, Dewey SL, Tsirka SE. A novel approach for imaging brain-behavior relationships in mice reveals unexpected metabolic patterns during seizures in the absence of tissue plasminogen activator. Neuroimage. 2007;38(1):34-42. https://doi.org/10.101 6/j.neuroimage.2007.06.032.

29. Bray NL, Pimentel H, Melsted P, Pachter L. Near-optimal probabilistic RNAseq quantification. Nat Biotechnol. 2016;34(5):525-7. https://doi.org/10.1038/ nbt.3519.

30. Melsted P, Ntranos V, Pachter L. The barcode, UMI, set format and BUStools. Bioinformatics. 2019;35(21):4472-3. https://doi.org/10.1093/bioinformatics/ btz279.
31. Satija R, Farrell JA, Gennert D, Schier AF, Regev A. Spatial reconstruction of single-cell gene expression data. Nat Biotechnol. 2015;33(5):495-502. https:// doi.org/10.1038/nbt.3192.

32. Lai L, Hennessey J, Bares V, Son EW, Ban Y, Wang W, et al. GSKB: A gene set database for pathway analysis in mouse. bioRxiv. 2016:082511. https://doi. org/10.1101/082511.

33. Quarantelli M, Berkouk K, Prinster A, Landeau B, Svarer C, Balkay L, et al. Integrated software for the analysis of brain PET/SPECT studies with partialvolume-effect correction. J Nucl Med. 2004;45(2):192-201.

34. Rousset OG, Ma Y, Evans AC. Correction for partial volume effects in PET: principle and validation. J Nucl Med. 1998;39:904-11.

35. Rask T, Dyrby T, Comerci M, Quarantelli M, Alfano B, Berkouk K, Baron J, Colchester A, Hojjat A, Knudsen GM: PVElab: Software for correction of functional images for partial volume errors. In Proceedings of the 10th Meeting of the Organisation for Human Brain Mapping. 2004

36. Passamonti L, Tsvetanov K, Jones P, Bevan-Jones WR, Arnold R, Borchert RJ, et al. Neuroinflammation and functional connectivity in Alzheimer's disease: interactive influences on cognitive performance. J Neurosci. 2019;39(36): 7218-26. https://doi.org/10.1523/JNEUROSCI.2574-18.2019.

37. Malpetti M, Kievit RA, Passamonti L, Jones PS, Tsvetanov KA, Rittman T, et al. Microglial activation and tau burden predict cognitive decline in Alzheimer's disease. Brain. 2020;143(5):1588-602. https://doi.org/10.1093/bra in/awaa088.

38. Orihuela R, McPherson CA, Harry GJ. Microglial M1/M2 polarization and metabolic states. Br J Pharmacol. 2016;173(4):649-65. https://doi.org/1 $0.1111 /$ bph.13139.

39. Dungan KM, Braithwaite SS, Preiser J-C. Stress hyperglycaemia. Lancet. 2009; 373(9677):1798-807. https://doi.org/10.1016/S0140-6736(09)60553-5.

40. Grabert K, Michoel T, Karavolos MH, Clohisey S, Baillie JK, Stevens MP, et al. Microglial brain region- dependent diversity and selective regional sensitivities to aging. Nat Neurosci. 2016;19(3):504-16. https://doi.org/10.103 8/nn.4222.

41. Gordon BA, Blazey TM, Su Y, Hari-Raj A, Dincer A, Flores S, et al. Spatial patterns of neuroimaging biomarker change in individuals from families with autosomal dominant Alzheimer's disease: a longitudinal study. Lancet Neurol. 2018;17(3):241-50. https://doi.org/10.1016/S1474-4422(18)30028-0.

42. McConathy J, Sheline YI. Imaging biomarkers associated with cognitive decline: a review. Biol Psychiatry. 2015;77(8):685-92. https://doi.org/10.1016/ j.biopsych.2014.08.024

43. Förster $\mathrm{S}$, Grimmer $\mathrm{T}$, Miederer I, Henriksen G, Yousefi BH, Graner $\mathrm{P}$, et al. Regional expansion of hypometabolism in Alzheimer's disease follows amyloid deposition with temporal delay. Biol Psychiatry. 2012;71(9):792-7. https://doi.org/10.1016/j.biopsych.2011.04.023.

44. Chételat $G$, Desgranges B, Landeau B, Mézenge F, Poline J, de La Sayette V, et al. Direct voxel-based comparison between grey matter hypometabolism and atrophy in Alzheimer's disease. Brain. 2008;131(Pt 1):60-71. https://doi. org/10.1093/brain/awm288.

45. Oblak AL, Lin PB, Kotredes KP, Pandey RS, Garceau D, Williams HM, et al. Comprehensive evaluation of the 5XFAD mouse model for preclinical testing applications: a MODEL-AD study. Front Aging Neurosci. 2021;13. https://doi.org/10.3389/fnagi.2021.713726.

46. Sierra A, Beccari S, Diaz-Aparicio I, Encinas JM, Comeau S, Tremblay M-Ė. Surveillance, phagocytosis, and inflammation: how never-resting microglia influence adult hippocampal neurogenesis. Neural Plast. 2014;2014:1-15. https://doi.org/10.1155/2014/610343.

47. Suárez-Calvet M, Kleinberger G, Caballero MÁA, Brendel M, Rominger A, Alcolea D, et al. sTREM2 cerebrospinal fluid levels are a potential biomarker for microglia activity in early-stage Alzheimer's disease and associate with neuronal injury markers. EMBO Mol Med. 2016;8(5):466-76. https://doi.org/1 0.15252/emmm.201506123.

48. Ewers M, Franzmeier N, Suárez-Calvet M, Morenas-Rodriguez E, Caballero MAA, Kleinberger G, et al. Increased soluble TREM2 in cerebrospinal fluid is associated with reduced cognitive and clinical decline in Alzheimer's disease. Sci Transl Med. 2019;11:eaav6221.

49. Rangaraju S, Dammer EB, Raza SA, Rathakrishnan P, Xiao H, Gao T, et al. Identification and therapeutic modulation of a pro-inflammatory subset of disease-associated-microglia in Alzheimer's disease. Mol Neurodegeneration. 2018;13(1):24. https://doi.org/10.1186/s13024-018-0254-8.

50. Yeh FL, Hansen DV, Sheng M. TREM2, microglia, and neurodegenerative diseases. Trends Mol Med. 2017;23(6):512-33. https://doi.org/10.1016/j. molmed.2017.03.008. 
51. Lee CD, Daggett A, Gu X, Jiang L-L, Langfelder P, Li X, et al. Elevated TREM2 gene dosage reprograms microglia responsivity and ameliorates pathological phenotypes in Alzheimer's disease models. Neuron. 2018;97: 1032-48 e1035.

52. Zhong L, Chen X-F, Wang T, Wang Z, Liao C, Wang Z, et al. Soluble TREM2 induces inflammatory responses and enhances microglial survival. J Exp Med. 2017;214(3):597-607. https://doi.org/10.1084/jem.20160844.

53. Zhong L, Xu Y, Zhuo R, Wang T, Wang K, Huang R, et al. Soluble TREM2 ameliorates pathological phenotypes by modulating microglial functions in an Alzheimer's disease model. Nat Commun. 2019;10:1-16.

54. Liu D, Cao B, Zhao Y, Huang H, McIntyre RS, Rosenblat JD, et al. Soluble TREM2 changes during the clinical course of Alzheimer's disease: A metaanalysis. Neurosci Lett. 2018;686:10-6. https://doi.org/10.1016/j.neulet.2018. 08.038 .

55. Suárez-Calvet M, Caballero MÁA, Kleinberger G, Bateman RJ, Fagan AM, Morris JC, et al. Early changes in CSF STREM2 in dominantly inherited Alzheimer's disease occur after amyloid deposition and neuronal injury. Sci Transl Med. 2016;8:369ra178.

\section{Publisher's Note}

Springer Nature remains neutral with regard to jurisdictional claims in published maps and institutional affiliations.

- fast, convenient online submission

- thorough peer review by experienced researchers in your field

- rapid publication on acceptance

- support for research data, including large and complex data types

- gold Open Access which fosters wider collaboration and increased citations

- maximum visibility for your research: over $100 \mathrm{M}$ website views per year

At $\mathrm{BMC}$, research is always in progress. 